\title{
The impact of age, ongoing task difficulty, and cue salience on preschoolers' prospective memory performance: The role of executive function
}

\author{
Caitlin E.V. Mahy $^{\mathrm{a}, \mathrm{b}, *}$, Louis J. Moses ${ }^{\mathrm{a}}$, Matthias Kliegel ${ }^{\mathrm{b}}$ \\ a Department of Psychology, 1227 University of Oregon, Eugene, OR 97403, USA \\ ${ }^{\mathrm{b}}$ Department of Psychology, University of Geneva, CH-1211 Geneva, Switzerland
}

\section{A R T I C L E I N F O}

\section{Article history:}

Available online 7 March 2014

\section{Keywords:}

Prospective memory

Cue salience

Ongoing task difficulty

Executive function

Inhibition

Preschoolers

\begin{abstract}
A B S T R A C T
The current study examined the impact of age, ongoing task (OT) difficulty, and cue salience on 4- and 5-year-old children's prospective memory (PM) and also explored the relation between individual differences in executive function (working memory, inhibition, and shifting) and PM. OT difficulty and cue salience are predicted to affect the detection of PM cues based on the multiprocess framework, yet neither has been thoroughly investigated in young children. OT difficulty was manipulated by requiring children to sort cards according to the size of pictured items (easy) or by opposite size (difficult), and cue salience was manipulated by placing a red border around half of the target cues (salient) and no border around the other cues (non-salient). The 5-year-olds outperformed the 4-year-olds on the PM task, and salient PM cues resulted in better PM cues compared with non-salient cues. There was no main effect of OT difficulty, and the interaction between cue salience and OT difficulty was not significant. However, a planned comparison revealed that the combination of non-salient cues and a difficult OT resulted in significantly worse PM performance than that in all of the other conditions. Inhibition accounted for significant variance in PM performance for non-salient cues and for marginally significant variance for salient cues. Furthermore, individual differences in inhibition fully mediated the effect of age on PM performance. Results are discussed in the context of
\end{abstract}

* Corresponding author at: Department of Psychology, 1227 University of Oregon, Eugene, OR 97403, USA.

E-mail address: cmahy@uoregon.edu (C.E.V. Mahy). 
the multiprocess framework and with reference to preschoolers' difficulty with the executive demands of dividing attention between the OT and PM task.

(c) 2014 Elsevier Inc. All rights reserved.

\section{Introduction}

How does a young boy remember to retrieve his art project from his teacher when he is engaged in a cognitively demanding task such as completing a complex puzzle? This ability to carry out intended activities in the future is defined as prospective memory (PM; Einstein \& McDaniel, 1990) and is an essential skill for young children to develop in order to establish independence from their parents and caregivers (e.g., Kvavilashvili, Messer, \& Ebdon, 2001; Meacham, 1982). PM develops markedly during early childhood (e.g., Kliegel \& Jäger, 2007; Kvavilashvili et al., 2001; Mahy \& Moses, 2011; but see Somerville, Wellman, \& Cultice, 1983), preparing children for carrying out tasks associated with school entry and allowing them to become increasingly responsible for fulfilling their intentions without external help. In the current study, we examined some of the factors that might be implicated in these developments within an executive function (EF) framework.

In a typical event-based PM laboratory paradigm, children must carry out an ongoing task (OT) while also remembering to perform a prospective action when they encounter a specific cue embedded within the OT (see Kvavilashvili et al., 2001). For successful PM, a child must appropriately divide attention between performing the OT and detecting the PM cues. Thus, a young girl who is engrossed in an ongoing activity not only must remember what she must do and when she must do it but also must disengage attention from the OT in order to detect PM cues (see Maylor, 1996).

Given the need to allocate attention to both the OT and cue detection (and to carrying out the PM task), such PM paradigms can be conceptualized as dual-task procedures that require significant executive resources (e.g., Bisiacchi, Schiff, Ciccola, \& Kliegel, 2009; Einstein, McDaniel, Smith, \& Shaw, 1998; Ihle, Hering, Mahy, Bisiacchi, \& Kliegel, 2013; Smith, 2003). If so, then preschool-aged children in particular may struggle with PM because they are known to struggle with EF more broadly (e.g., Anderson, 2002; Hongwanishkul, Happaney, Lee, \& Zelazo, 2005; Jones, Rothbart, \& Posner, 2003; Rueda, Rothbart, McCandliss, Saccomanno, \& Posner, 2005; Zelazo, Carlson, \& Kesek, 2008; Zelazo, Carter, Reznick, \& Frye, 1997). Substantial developmental improvements occur during this period in EFs such as working memory, inhibition, shifting, and planning (e.g., Carlson, 2005). Moreover, studies that use dual-task procedures have documented 3- to 8-year-old children's difficulty in allocating executive resources to two tasks simultaneously (e.g., Gordon \& Olson, 1998; Halford, Maybery, \& Bain, 1986; Irwin-Chase \& Burns, 2000). Because PM paradigms involve dual tasks (PM and OT), executive abilities could play an important role in PM in young children and should be associated with age-related improvement in PM. The current study set out to test this hypothesis, extending previous work on PM in young children suggesting a role for EF in the development of PM (Ford, Driscoll, Shum, \& Macaulay, 2012; Mahy \& Moses, 2011).

Taking a task analysis approach, dividing resources between the OT and PM task requires inhibition to pull attention away from the one task, set shifting to switch flexibly between the two tasks, and working memory to keep in mind the rules for each of the tasks. Thus, EFs such as working memory, inhibition, and shifting are candidate developmental mechanisms of PM. Conceptual support for this task analysis comes from the multiprocess framework of PM (Einstein \& McDaniel, 2005; McDaniel \& Einstein, 2000). This framework suggests that both controlled and automatic processes can contribute to PM and that controlled, executive processes are more likely to be required under cognitively demanding conditions. The multiprocess framework suggests that characteristics of the ongoing task and the PM cue affect whether such controlled processes are necessary for PM retrieval (McDaniel \& Einstein, 2000). For example, controlled processes may be necessary for PM in the context of a demanding OT but may be less critical in the context of an easy OT. Working memory and inhibition 
may be involved in holding the OT instructions in mind as well as in performing a complex OT. These processes consume executive resources that could otherwise be used to detect PM cues. In addition, executive resources may be differentially taxed as a function of the salience of the PM cues. With salient cues, PM may rely more on automatic or lower level perceptual processes rather than executive processes. A salient PM cue may simply "pop out" of the environment (see Einstein, McDaniel, Manzi, Cochran, \& Baker, 2000) and, therefore, put less demand on controlled processes in order for the cue to be detected. However, controlled processes are likely involved in cue detection and intention retrieval to some extent, such that even in the face of minimal demands on EF effortful control is probably recruited, although to a lesser extent than when demands on EF are high. If preschool-aged children struggle with dividing attention and sharing executive resources between two tasks, then manipulations that support shifting between the OT and PM task (e.g., an easy OT, salient PM cues) should enhance PM, whereas manipulations that disrupt this shifting (e.g., a demanding OT, non-salient PM cues) should result in lower PM performance.

A number of studies have attempted to manipulate the executive demands of PM tasks. With respect to the OT, several studies show that for school-aged children more difficult OTs generally lead to worse PM performance than easier OTs (Kliegel et al., 2013; but see Ward, Shum, McKinlay, BakerTweney, \& Wallace, 2005). To our knowledge, only two studies have targeted preschool children. Kvavilashvili and colleagues (2001) compared preschoolers' PM in a condition where the OT needed to be interrupted in order to carry out the prospective intention with a condition where the prospective intention was carried out immediately after the completion of the OT. This can be thought of as an especially strong manipulation of OT difficulty (i.e., presence vs. absence of an OT at the time the prospective cue appears). Children's PM was significantly worse when they were required to interrupt the OT to carry out the prospective intention.

In the only other study with preschoolers, Wang, Kliegel, Liu, and Yang (2008) compared PM performance in a condition where children were asked to memorize OT items with that in a condition where they were not asked to do so. The need to memorize OT items resulted in slower PM response times, but it did not influence PM accuracy. In this study, however, the extent to which children actually followed the instructions to memorize items is unknown because there was no objective measure of OT performance to confirm that memorization was actually associated with difficulty of the OT. Hence, it is not clear from this study whether OT memory load truly had little impact on PM or whether the manipulation of difficulty was simply not effective. Moving forward, an essential feature of future studies examining the impact of OT difficulty on PM is an objective measure of OT performance in order to confirm that the manipulation of difficulty was in fact successful.

With respect to manipulations of cue salience, McGann, Defeyter, Ellis, and Reid (2005) found that in school-aged children larger PM cues (in terms of perceptual size) relative to OT stimuli resulted in better PM than cues that were the same size as the OT items. Similarly, Kliegel and colleagues (2013) found that lower levels of perceptual salience (a single flowerpot as a cue) resulted in worse PM in school-aged children compared with cues that were more salient (several flowerpots as a cue). Therefore, it seems that high cue salience can be quite effective in aiding children's PM performance by boosting the likelihood of cue detection. It is not yet known, however, whether manipulations of salience affect PM in preschool-aged children.

In sum, these studies establish that OT difficulty and cue salience affect school-aged children's PM, but they have not been thoroughly investigated within the critical preschool period, a time when divided attention tasks may pose a particular challenge to children's limited executive resources (e.g., Gordon \& Olson, 1998). Moreover, to the best of our knowledge, no studies have manipulated these two factors within the same experiment (to examine whether their effects are independent or interactive). Because performance of the OT and detection of the PM cue may rely on similar or shared executive resources, conditions under which OT difficulty is high and cue salience is low may pose the greatest difficulties for PM performance, especially in preschoolers with their limited executive resources. That is, PM performance may be affected by ongoing task difficulty and cue salience in more than simple additive ways. Consequently, the primary aim of the current study was to assess the independent and possibly interactive effects of OT difficulty and cue salience in preschoolers.

A secondary aim of this study was to assess the role of individual differences in EF in PM performance. During the late preschool period between 4 and 5 years of age, children show qualitative 
and quantitative changes in executive control (e.g., Carlson, 2005; Espy, 1997; Perner \& Lang, 1999; Zelazo, Müller, Frye, \& Marcovitch, 2003) that may affect PM development in this age range specifically. To the best of our knowledge, only two studies have investigated the relation between EF and PM in preschool children. Mahy and Moses (2011) found that working memory but not inhibition predicted 4- to 6-year-olds' PM, whereas Ford and colleagues (2012) found that inhibitory control but not working memory predicted 4- to 6-year-olds' PM. Although these studies established relations among preschoolers' PM and EF, they present conflicting findings and, moreover, did not examine the role of EF in the age effect on PM. Therefore, a secondary goal of the current study was to further examine individual differences in EF and relate them to age effects on PM.

\section{The current study}

The current study manipulated OT difficulty (between participants) and cue salience (within participants) in a PM paradigm with 4- and 5-year-old children to examine the impact of varying levels of executive demand on retrieval processes in PM. Children were assigned to either an easy OT or a difficult OT, where they received half salient PM cues and half non-salient PM cues. We also measured individual differences in EF (working memory, inhibition, and set shifting; see Miyake et al., 2000) and examined their relation with PM performance as well as whether they mediated age effects in PM.

Our hypotheses were as follows:

1. Five-year-olds will outperform 4-year-olds on the PM task, based on past studies showing developmental differences in PM in this age range (e.g., Ford et al., 2012; Mahy \& Moses, 2011) and the advances in EF that occur between 4 and 5 years of age specifically (e.g., Carlson, 2005).

2. A difficult OT will result in worse PM compared with an easy OT.

3. Salient cues will result in better PM than non-salient cues.

4. A difficult OT and non-salient PM cues will result in by far the lowest PM performance compared with the other three conditions because this combination of factors would impose a particularly high demand on executive resources (i.e., an interactive effect rather than merely additive).

5. Individual differences in EF, particularly inhibition and working memory (given the findings of Ford et al., 2012, and Mahy \& Moses, 2011), will predict PM performance and may account for the age effect in PM.

\section{Method}

\section{Participants}

The participants were 1194 - and 5-year-olds (53 girls and 66 boys). Among these children, 7 were not included in the final analysis: 6 for failing the control question in the PM task and 1 for failing to reach criterion in the practice trials of the card sorting component of the PM task. The final sample consisted of 112 children: 56 4-year-olds ( $M=52.98$ months, $S D=3.56 ; 28$ girls and 28 boys) and 56 5-year-olds $(M=66.14$ months, $S D=3.88 ; 22$ girls and 34 boys). All children met the criterion for normal verbal ability, scoring at least 85 (1 standard deviation below the standardized mean) on the Peabody Picture Vocabulary Test (PPVT-III; Dunn \& Dunn, 1997). Children were randomly assigned to the easy or difficult OT condition. Mostly Caucasian children (84\% were Caucasian, $8 \%$ had Native American or mixed ethnic backgrounds, and $8 \%$ declined to report ethnicity) from middle-class backgrounds participated, reflecting the population from which the sample was taken. Participants were recruited from a university database compiled from birth announcements in local newspapers.

\section{Measures}

\section{Prospective memory paradigm}

Ongoing task. For the OT, children were asked to help a family who had just moved into a new house and needed help sorting their things into categories of small or large items. Children were asked to name whether an item depicted on a card was small or large and then to place the card into the 
appropriate box on the table in front of them. The manipulation of OT difficulty was between participants, such that half of the children received a difficult card-sorting task and half received an easy one. Children were asked to sort cards into boxes that had an exemplar of the category on the front. In the easy condition, they needed to sort cards into the corresponding box (e.g., large cards into the box with a large item on it, small cards into the box with a small item on it). In the difficult condition, they were asked to sort the cards in a "silly" way into opposite categories (e.g., large cards into the box with a small item on it, small cards into the box with a large item on it). The difficult condition was designed to have higher demands on (a) working memory, because children could not simply rely on the exemplars on the box to aid their sorting but also needed to remember the rule to sort by opposites, and (b) inhibition, because children needed to overcome the prepotent response to place the card in the box with a matching exemplar.

Household items were depicted on cards that were approximately 3 by 3 inches. Large items were larger than small items both in the real world and in pictorial size. The large items took up most of the space on the cards, whereas the small items took up a small square in the middle of the card (approximately 1 by 1 inch; see Fig. 1). Children practiced naming and then sorting five cards depicting the household items according to size to ensure that they understood the rules. Only children who were able to sort at least four of five cards correctly were included in the analyses. As noted earlier, only one child did not meet this criterion for card sorting.

OT performance was measured by assessing children's accuracy in sorting 96 cards into the appropriate boxes as well as the number of incorrect initial reaches that children made in card sorting. For each card sorted, children were given two points for sorting the card correctly on the first attempt, 1 point for initially reaching to the incorrect box and then correcting themselves to place it into the correct box, and 0 points for placing the card in the incorrect box. Thus, children's OT score was out of a possible 192 points because children eventually sorted all cards into small and large categories (including the PM cue cards).

Prospective memory task. In addition to card sorting, children were told that the family members were also looking for their pet animals (PM instruction). They were asked to help the family by ringing a bell on a table approximately three feet behind them if they saw an animal pictured on a card. This action was selected based on pilot testing indicating that ringing a bell was interesting and motivating to children of this age. After children were asked whether they understood the rules, following standard procedures in PM research (see Kvavilashvili, Kyle, \& Messer, 2008), they drew pictures for a 3-min delay period to allow some time for forgetting. After this delay interval, children began the card sorting. Cards were divided into three stacks of 32, each of which contained two PM cue cards. Cue cards depicted a guinea pig, a dog, a goldfish, a cat, a parrot, and a rabbit. The cue cards appeared as the 12th and 30th cards in the first stack, the 14th and 21st cards in the second stack, and the 7th and 23rd cards in the third stack. In between sets of cards, children drew pictures for 1 min. The PM score was the number of times that children rang the bell when they encountered an animal card (0-6).

Cue salience was manipulated within participants, such that each child received three PM cue cards with a salient red border and three PM cue cards that were non-salient without a colored border (see Fig. 2). Thus, the cues with a colorful border were more distinct from the OT items than the cards with
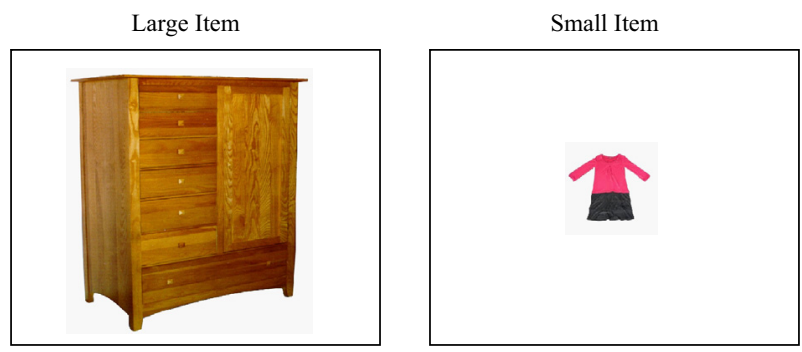

Fig. 1. Example of ongoing task stimuli. 

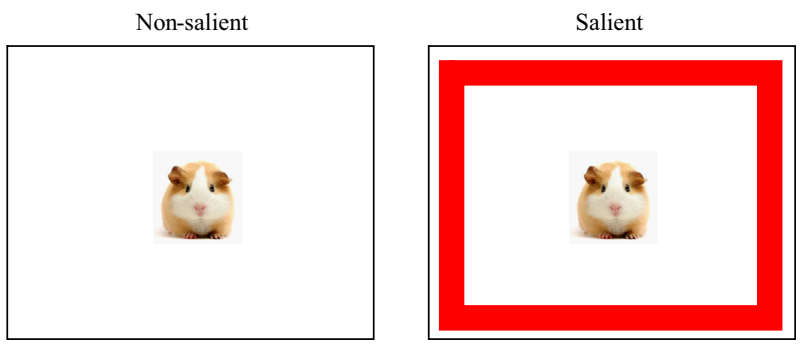

Fig. 2. Example of salient and non-salient prospective memory cues.

no border. Whether an individual cue card had a border or not was based on a random order for each child drawn from all 20 possible orders with the restriction that three had borders and three had no borders.

As a control, at the end of the PM task, all children were asked, "What were you supposed to do in this game when you saw a picture of an animal?" Only children who answered this question correctly were included in the final analysis in order to rule out PM errors due to retrospective memory failures. As noted earlier, six children failed to answer this question correctly.

\section{Backward Digit Span}

To measure working memory, children completed the digits forward and digits backward subscales from the Wechsler Intelligence Scale for Children-third edition (WISC-III; Wechsler, 1991). The Backward Digit Span task was used as the measure of working memory, but the Forward Digit Span was also administered as a warm-up and to familiarize children with the task. In the Backward Digit Span task, children were asked to repeat a series of numbers in backward order after the experimenter read them aloud. They began with two numbers, and after completing two trials successfully an additional number was added. The task ended when children failed on two consecutive trials. Backward Digit Span was calculated by summing the number of backward digit strings children were able to repeat accurately.

\section{Simon Says}

The Simon Says task was used as a measure of conflict inhibition (Carlson, 2005). The experimenter and children stood facing each other, and the experimenter asked children to do some silly things such as "touch your feet." Then the experimenter explained that children should perform an action only if she prefaced the command with "Simon says." Children were told to remain perfectly still otherwise. They were given two practice trials in which they were asked to show the experimenter what they would do if she said "Simon says clap your hands" and "clap your hands". If children performed either of the practice trials incorrectly, the experimenter told them what the correct response should be. The experimenter then issued commands in quick succession without demonstrating the actions. A total of 10 trials were administered ( 5 with and 5 without "Simon says"). Performance on non-Simon-says trials was taken as an index of inhibitory ability $(0=$ commanded movement, $1=$ partial movement, 2 = different movement, 3 = no movement; scored individually for each non-Simon-says trial; range $=0-15$; Carlson \& Meltzoff, 2008). Interrater reliability on non-Simon-says trials was high $(r>.85)$.

\section{Card Sort}

The Dimensional Change Card Sort was used a measure of set shifting (Frye, Zelazo, \& Palfai, 1995). Children were shown two boxes with target cards (e.g., a blue rabbit and a red boat) on the front of them. The experimenter presented a series of cards (red and blue rabbits and boats) and instructed children to place all of the rabbits in the box with the red rabbit and to place all of the boats in the box with the blue boat in the "shape game." Children were given two practice trials in which they received feedback on their performance. Then they completed five test trials. After children had sorted 
five cards in the shape game, the experimenter announced that they were not going to play the shape game and would now play the "color game." Children were told that in the color game all of the red items should go in the box with the red boat on it and all of the blue items should go in the box with the blue rabbit on it. There were five post-switch trials, and three of these trials were incompatible with the first rule (conflict trials where sorting by the old rule would now lead to an incorrect response; see Marcovitch, Boseovski, \& Knapp, 2007). Before each of the 10 trials, the rule and the relevant dimension were identified by the experimenter (e.g., "Remember, red cards go here and blue cards go here. Here's a red boat") in order to avoid errors due to working memory failures, and then the card was given to children to sort. The total number of correct incompatible post-switch trials was recorded (0-3).

\section{Peabody Picture Vocabulary Test}

Children completed the PPVT-III (Dunn \& Dunn, 1997) as a measure of verbal ability. They were asked to point to the picture corresponding to the word the experimenter read aloud. For each word, children were presented with four line drawings from which to choose. They were given two practice trials in which they were given feedback and told that it was okay to guess if they did not know what the word meant. Then children were given the first set of words depending on their chronological age. The test was administered until participants failed 8 of 12 items in a given set. Raw scores on this measure were used in the analysis rather than age-corrected scores.

\section{Procedure}

Children were interviewed individually by an experimenter in a university laboratory, and all tasks were administered in a fixed order (as is convention in individual differences research): PM task, Backward Digit Span, Simon Says, Card Sort, and PPVT-III. Parents were asked to provide demographic information while their children participated in the study. At the end of the experimental session, children were given a small toy and parents were given $\$ 10$ (U.S.) and were thanked for their participation. The university ethics review board approved all procedures.

\section{Results}

Ongoing task difficulty manipulation check

To assess whether the manipulation of OT difficulty was successful, a 2 (Age: 4 - vs. 5 -year-olds) $\times 2$ (OT difficulty: easy vs. difficult) analysis of variance (ANOVA) was conducted on OT accuracy (card sorting). OT accuracy was significantly affected by age and OT difficulty. The 5-year-olds did better on the OT $(M=180.19, S D=9.37)$ than the 4-year-olds $(M=170.09, S D=31.02), F(1,103)=5.26$, $p=.02, \eta^{2}=.05$. In addition, children in the difficult condition performed worse on card sorting $(M=168.95, S D=30.81)$ than those in the easy condition $(M=181.60, S D=7.36), F(1,103)=8.50$, $p=.004, \eta^{2}=.08$. The interaction between age and OT difficulty was not significant $(p=.26$, $\eta^{2}=.01$ ). Thus, the manipulation of task difficulty in the OT was considered as successful and did not differentially affect 4 - and 5-year-olds' OT performance.

\section{Prospective memory task}

Table 1 shows means of PM performance by age, OT difficulty, and cue salience. A 2 (Age: 4- vs. 5 -year-olds) $\times 2$ (OT difficulty: easy vs. difficult) $\times 2$ (Cue Salience: salient vs. non-salient cues) mixed ANOVA on PM performance with repeated measures on the last factor revealed a significant main effect of age. The 5-years-olds $(M=2.80, S D=2.29)$ performed better than the 4-year-olds $(M=1.70$, $S D=2.15)$ on the PM task, $F(1,108)=6.92, p=.01, \eta^{2}=.06$. There was no significant effect of OT difficulty on PM $\left(p=.31, \eta^{2}=.01\right)$. However, children remembered to carry out the PM task more often for salient cues $(M=1.29, S D=1.33)$ compared with non-salient cues $(M=0.96, S D=1.15)$, 
Table 1

Means and standard deviation of prospective memory performance by condition.

\begin{tabular}{|c|c|c|c|c|}
\hline & \multicolumn{2}{|c|}{$\underline{\text { Easy ongoing task }}$} & \multicolumn{2}{|c|}{$\underline{\text { Difficult ongoing task }}$} \\
\hline & Salient & Non-salient & Salient & Non-salient \\
\hline 4-year-olds & $1.00(1.36)$ & $0.79(1.07)$ & $1.07(1.33)$ & $0.54(0.88)$ \\
\hline 5-year-olds & $1.68(1.34)$ & $1.46(1.29)$ & $1.43(1.23)$ & $1.04(1.17)$ \\
\hline Total & $1.34(1.38)$ & $1.13(1.22)$ & $1.25(1.28)$ & $0.79(1.06)$ \\
\hline
\end{tabular}

$F(1,108)=13.25, p<.001, \eta^{2}=.11$. There were no significant interactions among age, OT difficulty, and cue salience.

A planned comparison revealed that performance in the difficult OT condition for non-salient cues ( $M=0.79, S D=1.06$ ) was worse than PM performance averaged across the three other conditions $(M=1.24, S D=1.29), t(56)=2.25, p=.03$, Cohen's $d=0.38$. Thus, PM was significantly worse under conditions of cue non-salience and a difficult OT.

\section{Individual differences in performance on executive tasks}

The three behavioral measures of EF-Backward Digit Span, Simon Says, and Card Sort-were highly intercorrelated, $r \mathrm{~s}(108)>.43$, ps < .001 (see Table 2). Importantly, these tasks had acceptable statistics, indicating that multicollinearity was not a problem (variance inflation factors [VIFs] $<1.92$, tolerances > 0.75). Because specific components of EF have been found to correlate with preschoolers' PM (e.g., Ford et al., 2012; Mahy \& Moses, 2011), however, scores on the three EF measures were examined individually as predictors of PM separately for salient and non-salient PM cues.

A first regression analysis predicted PM performance for salient cues from children's age in months (first step), PPVT-III score (second step), and Backward Digit Span, Simon Says, and Card Sort (third step). Performance on Simon Says was the only marginally significant predictor of PM performance for salient cues in the full model (see Table 3). Age, verbal ability, and EF accounted for $14.2 \%$ of the variance in PM for salient cues. Performance on the EF tasks did not explain a significant amount of variance above and beyond age and verbal ability, $R^{2}$ change $=.05, F(3,102)=2.04, p=.11$.

In a corresponding second regression analysis for non-salient cues, performance on the Simon Says task independently predicted PM performance for non-salient cues (see Table 3 ). In addition, PPVT-III score marginally predicted PM performance. Age, verbal ability, and EF together accounted for $22.6 \%$ of the variance in PM performance for non-salient cues. Performance on the EF tasks added significantly to the prediction of PM performance above and beyond age for non-salient cues, $R^{2}$ change $=.08$, $F(3,102)=3.57, p=.017$.

Because inhibition, as measured by the Simon Says task, was the best predictor of PM performance for non-salient cues and a marginally significant predictor for salient cues, we sought to investigate whether inhibition might account for the effect of age on PM (Fig. 3). We used a mediation analysis with bootstrapping (following Preacher \& Hayes, 2008) to examine whether Simon Says performance mediated the relation between age and PM performance. Age significantly predicted both PM

\section{Table 2}

Correlations among behavioral measures.

\begin{tabular}{|c|c|c|c|c|}
\hline & 1 & 2 & 3 & 4 \\
\hline \multicolumn{5}{|l|}{ 1. Age } \\
\hline 2. Prospective memory & $.28^{* *}$ & & & \\
\hline 3. Backward Digit Span & $.44^{* *}$ & $.28^{* *}\left(.16^{\dagger}\right)$ & & \\
\hline 4. Simon Says & $.50^{* *}$ & $.40^{* *}\left(.32^{* *}\right)$ & $.61^{* *}\left(.51^{* *}\right)$ & \\
\hline 5. Card Sort & $.28^{* *}$ & $.32^{* *}\left(.25^{* *}\right)$ & $.44^{* *}\left(.35^{* * *}\right)$ & $.43^{* *}\left(.36^{* *}\right)$ \\
\hline
\end{tabular}

Note. Partial correlations, controlling for age in months, are in parentheses. Degrees of freedom range from 105 to 112 .

$+p<.10$.

${ }^{* * *} p<.01$. 
Table 3

Regression analyses for salient and non-salient cues.

\begin{tabular}{|c|c|c|c|c|c|c|c|}
\hline & & \multicolumn{3}{|c|}{ Salient PM cues } & \multicolumn{3}{|c|}{ Non-salient PM cues } \\
\hline & & $B$ & $S E$ & $\beta$ & B & $S E$ & $\beta$ \\
\hline Step 1 & Age & .039 & .017 & $.222^{*}$ & .040 & .014 & $.267^{* *}$ \\
\hline Step 2 & PPVT-III & .019 & .009 & $.231^{*}$ & .022 & .007 & $310^{* *}$ \\
\hline \multirow[t]{5}{*}{ Step 3} & Age & .003 & .020 & .018 & .003 & .016 & .022 \\
\hline & PPVT-III & .012 & .009 & .150 & .015 & .008 & $.211^{\dagger}$ \\
\hline & Working memory & -.029 & .119 & -.029 & -.082 & .098 & -.097 \\
\hline & Inhibition & .059 & .030 & $.247^{\dagger}$ & .058 & .025 & $.284^{*}$ \\
\hline & Set shifting & .087 & .118 & .079 & .155 & .097 & .162 \\
\hline
\end{tabular}

$+p<.10$.

${ }^{*} p<.05$.

*** $p<.01$

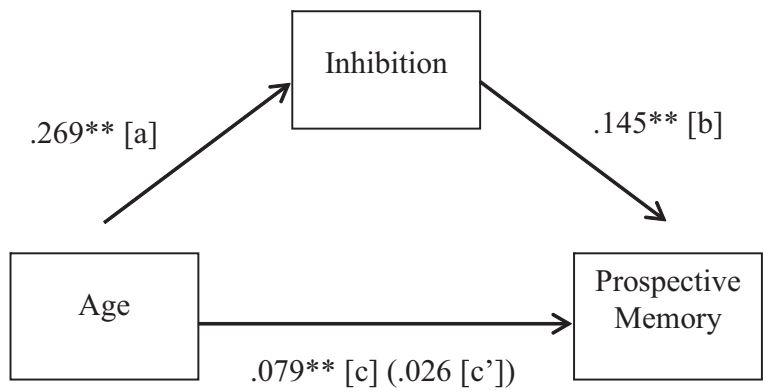

Fig. 3. Inhibition as a mediator of the association between age and prospective memory performance. The effect of the age on the inhibition is path [a]. The effect of inhibition on prospective memory performance, controlling for age, is path [b]. Path [c] represents the effect of age on prospective memory performance. Path $\left[\mathrm{c}^{\prime}\right]$ represents the effect of age on the prospective memory after including inhibition in the model. The coefficient for path $\left[\mathrm{c}^{\prime}\right]$ is in parentheses. ${ }^{* *} p<.01$.

performance (path [c] in Fig. 3) and inhibition (path [a]). However, when the link between inhibition and PM (path [b]) was added, age was no longer a significant predictor of PM (path [ $\left.\mathrm{c}^{\prime}\right]$ ). These results suggest no direct association between age and PM but that this association is fully mediated by developmental changes in children's inhibition. Thus, this provides preliminary evidence suggesting that inhibition may be an important mechanism contributing to age-related increases in PM during early development.

\section{Discussion}

The current study took a two-pronged approach to studying the relation between EF and PM in preschoolers. A task manipulation approach examined the impact of age, OT difficulty, and cue salience on preschoolers' PM. Several important findings emerged. First, 5-year-olds had better PM than 4year-olds, consistent with previous studies (Ford et al., 2012; Mahy \& Moses, 2011). Second, children's PM was better for salient cues compared with non-salient cues. Third, although children's OT performance was worse in the difficult OT condition compared with the easy OT condition, OT difficulty had no significant impact on PM performance. Finally, comparing the most difficult condition (difficult OT, non-salient cues) with the other conditions revealed that PM performance was significantly lower in the most challenging condition. An individual differences approach revealed that EF scores were positively related to PM. Inhibition independently predicted PM for non-salient cues and marginally predicted PM for salient cues. A mediation analysis revealed that inhibition fully mediated the relation between age and PM, suggesting that it may be a critical mechanism of PM development in preschool children. 
The age-related increases documented in this study support the idea that developmental changes in PM are occurring rapidly during early childhood, such that age differences in performance are observable in narrow age ranges, such as between 4 and 5 years of age (for similar findings, see Ford et al., 2012; Mahy \& Moses, 2011, 2013). However, the results of the mediation analysis revealed that the effect of age on PM was no longer significant after adding inhibition as a mediator. Thus, it seems that inhibition may be implicated in age-related increases in PM during preschool, as past work has suggested (e.g., Kvavilashvili et al., 2001; Wang et al., 2008).

The robust effect of cue salience supports the idea that PM is strongly influenced by manipulations surrounding the PM cue (Kliegel et al., 2013; McGann et al., 2005). Similar to our findings, McGann and colleagues (2005) found that older children had better PM when the cues were perceptually larger than the OT items. Automatic processes may have been involved in the detection of salient PM cues so that cue detection was less effortful with highly noticeable cues (in line with predictions of the multiprocess framework; McDaniel \& Einstein, 2000). Consistent with this possibility, we found that EF contributed to the prediction of PM only for non-salient cues. However, this is not to suggest that cue detection relied solely on automatic processes because we would have expected higher levels of PM performance if this had been the case. To the contrary, the current study had fairly low levels of PM performance overall, suggesting that well-developed controlled processes may be necessary for detecting even salient PM cues.

Although the manipulation of OT difficulty was successful (i.e., it had an impact on OT performance), OT difficulty did not affect children's PM performance collapsing across cue salience levels. Importantly, however, OT difficulty did have an impact when cues were non-salient, suggesting that the effect of OT difficulty is more detrimental when combined with low levels of cue salience. The lack of a main effect of OT difficulty is consistent with some literature showing that certain manipulations of the OT have no impact on children's or adults' PM (e.g., Otani et al., 1997; Wang et al., 2008; Ward et al., 2005). There are two possible explanations for why no main effect of OT difficulty on PM was found. First, it is possible that controlled processing necessary for the OT has only a limited effect on PM cue detection because the two tasks may rely on (partly) different processes. Specifically, detection of PM cues (under specific conditions) may be to some extent an automatic process, such that manipulating the level of controlled processes necessary in the OT (via a manipulation of OT difficulty) may have limited impact on cue detection. If PM cues automatically trigger the prospective intention, at least under specific conditions, then effortful shifting of attention away from the OT to detect the PM cue might be unnecessary. A second possibility is that the difficulty level of the OT in our study was not sufficient to disrupt cue detection substantially. The difficult OT was certainly sufficient to reduce accuracy on card sorting, but it might not have been demanding enough to completely capture children's attention to prevent them from detecting the PM cues (particularly salient cues). Therefore, perhaps only highly cognitively demanding OTs interfere with executive processes necessary for the detection of PM cues. Future work should further investigate the effect of OT difficulty on the PM of preschool-aged children by using stronger manipulations of OT difficulty (e.g., see Kliegel et al., 2013).

There was no formal interaction between cue salience and OT difficulty. A comparison was carried out to investigate our prediction that the lowest PM performance would result from the difficult OT condition and non-salient cues. Our prediction was confirmed; PM performance was lowest for non-salient cues in the difficult OT condition compared with the three other conditions (both salient and non-salient cues in the easy OT condition and salient cues in the difficult OT condition). This finding suggests that while attention is captured by a challenging ongoing activity, perceptually non-salient cues are detrimental to PM because they do not provide clear signals to shift one's attention from the OT to the PM cue.

Inhibition predicted PM performance marginally for salient cues and significantly for non-salient cues. In past literature, inhibition has been cited as a necessary process in order to detect any PM cue and has been found to predict PM using a composite score of inhibition measures (see Ford et al., 2012). However, the current findings conflict with previous findings by Mahy and Moses (2011), who found that inhibition as measured by performance on a Stroop-like Day/Night task did not predict 4- to 6-year-olds' PM performance. Importantly, differences between the results of Mahy and Moses and those of the current study may be due to the limited variance produced by the Day/ Night task in the former study. Children were close to ceiling on the task, making it difficult to pick 
up on developmental changes in inhibitory control. In contrast, the Simon Says task used in the current study captures more variance in performance in this age range (see Carlson, 2005). That said, future studies should use more than one measure of inhibition in order to better examine the role of early developing inhibitory control in PM performance.

The important role of inhibition in explaining age effects in PM uncovered by the current study is in line with work with older adults showing that inhibition was a significant predictor of PM performance (Schnitzspahn, Stahl, Zeintl, Kaller, \& Kliegel, 2013). Inhibition fully mediated the relation between age and PM, supporting the idea that inhibition is one important mechanism driving PM development in this age range (Ford et al., 2012; Kvavilashvili et al., 2001). Overall, the individual differences results support the idea that EFs, especially inhibition, play an important role in PM, particularly when cues are non-salient (Einstein \& McDaniel, 1990; Martin, Kliegel, \& McDaniel, 2003; McDaniel \& Einstein, 2000).

From a theoretical perspective, the findings surrounding the role of inhibition, especially in PM with non-salient cues, provide support for the multiprocess framework because different levels of PM cue salience are differentially related to executive processes. Given that preschool-aged children have limited executive abilities (Carlson, 2005; Zelazo et al., 2003), salient cues may likely aid PM, particularly for young children who are engaged in a difficult ongoing task, by pulling their attention away from the OT. Without salient cues, however, it seems that children are often unable to inhibit the prepotent response to treat the PM cue like an OT item, supporting work indicating that 5-yearolds still struggle with dual tasks that require divided attention (Halford et al., 1986; Irwin-Chase \& Burns, 2000).

In contrast to children's performance on PM paradigms in past studies, children's PM performance in the current study was low overall; children carried out the PM intention on average only approximately a third of the time, suggesting that performing both an OT and detecting cues (a dual task) is challenging, perhaps due its high demands on executive resources. It is possible that both the easy and difficult OTs were in fact fairly difficult for children for three reasons. First, the PM task required a different level of analysis than the OT (i.e., PM cues required analysis of identity of the item, whereas OT performance required assessment of the size of the item; see Einstein et al., 2005; Kliegel, Jäger, \& Phillips, 2008). Second, the animals (PM cues) could be sorted by their size category and, thus, were easy for children to overlook as PM cues. Third, unlike previous paradigms that required card naming only, the current task required both size naming (big or small) and sorting.

In sum, this study is the first to examine both the impact of OT difficulty and PM cue salience (based on predictions of the multiprocess framework) on preschoolers' PM. Specifically, salient cues improved PM, suggesting that attention shifting between the OT and PM task was easier with a salient perceptual cue. Children's PM especially suffered under conditions of low PM cue salience and a difficult OT task. EF contributed significantly to PM performance for non-salient cues. In addition, inhibition fully mediated the age effect in PM. Future work should continue to investigate the mechanisms involved in PM development and factors that influence its performance, particularly during this critical preschool period when children must learn to complete some of their intentions independent of caregivers before entering school.

\section{Acknowledgments}

The authors acknowledge the Natural Sciences and Engineering Research Council of Canada and the Swiss Federal Commission for funding portions of this research with scholarships to C.E.V.M. and the German Research Foundation (DFG) for support provided to M.K. We thank Alexis White, Emily Collins, Katie Avery, and Martha Lopez for their research assistance as well as the families and children who participated.

\section{References}

Anderson, P. (2002). Assessment and development of executive function during childhood. Child Neuropsychology, 8, 71-82.

Bisiacchi, P. S., Schiff, S., Ciccola, A., \& Kliegel, M. (2009). The role of dual-task and task-switch in prospective memory: Behavioural data and neural correlates. Neuropsychologia, 47, 1362-1373. 
Carlson, S. M. (2005). Developmentally sensitive measures of executive function in preschool children. Developmental Neuropsychology, 28, 595-616.

Carlson, S. M., \& Meltzoff, A. N. (2008). Bilingual experience and executive functioning in young children. Developmental Science, $11,282-298$.

Dunn, L. M., \& Dunn, L. M. (1997). Peabody picture vocabulary test-Third edition. Circle Pines, MN: American Guidance Service.

Einstein, G. O., \& McDaniel, M. A. (1990). Normal aging and prospective memory. Journal of Experimental Psychology: Learning, Memory, and Cognition, 16, 717-726.

Einstein, G. O., \& McDaniel, M. A. (2005). Prospective memory: Multiple retrieval processes. Current Directions in Psychological Science, 14, 286-290.

Einstein, G. O., McDaniel, M. A., Manzi, M., Cochran, B., \& Baker, M. (2000). Prospective memory and aging: Forgetting intentions over short delays. Psychology and Aging, 15, 671-683.

Einstein, G. O., McDaniel, M. A., Smith, R. E., \& Shaw, P. (1998). Habitual prospective memory and aging: Remembering intentions and forgetting actions. Psychological Science, 9, 284-288.

Einstein, G. O., McDaniel, M. A., Thomas, R., Mayfield, S., Shank, H., Morrisette, N., et al (2005). Multiple processes in prospective memory retrieval: Factors determining monitoring versus spontaneous retrieval. Journal of Experimental Psychology: General, 134, 327-342.

Espy, K. A. (1997). The shape school: Assessing executive function in preschool children. Developmental Neuropsychology, 13, 495-499.

Ford, R. M., Driscoll, T., Shum, D., \& Macaulay, C. E. (2012). Executive and theory-of-mind contributions to event-based prospective memory in children: Exploring the self-projection hypothesis. Journal of Experimental Child Psychology, 111, 468-489.

Frye, D., Zelazo, P. D., \& Palfai, T. (1995). Theory of mind and rule-based reasoning. Cognitive Development, 10, 483-527.

Gordon, A. C., \& Olson, D. R. (1998). The relation between acquisition of a theory of mind and the capacity to hold in mind. Journal of Experimental Child Psychology, 68, 70-83.

Halford, G. S., Maybery, M. T., \& Bain, J. D. (1986). Capacity limitations in children's reasoning: A dual-task approach. Child Development, 57, 616-627.

Hongwanishkul, D., Happaney, K. R., Lee, W., \& Zelazo, P. D. (2005). Hot and cool executive function: Age-related changes and individual differences. Developmental Neuropsychology, 28, 617-644.

Ihle, A., Hering, A., Mahy, C. E. V., Bisiacchi, P. S., \& Kliegel, M. (2013). Adult age differences, response management, and cue focality in event-based prospective memory: A meta-analysis on the role of task order specificity. Psychology and Aging, 28, 714-720.

Irwin-Chase, H., \& Burns, B. (2000). Developmental change in children's abilities to share and allocate attention in a dual task. Journal of Experimental Child Psychology, 77, 61-85.

Jones, L. B., Rothbart, M. K., \& Posner, M. I. (2003). Development of executive attention in preschool children. Developmental Science, 6, 498-504.

Kliegel, M., \& Jäger, T. (2007). The effects of age and cue-action reminders on event-based prospective memory performance in preschoolers. Cognitive Development, 22, 33-46.

Kliegel, M., Jäger, T., \& Phillips, L. H. (2008). Adult age differences in event-based prospective memory: A meta-analysis on the role of focal versus nonfocal cues. Psychology and Aging, 23, 203-208.

Kliegel, M., Mahy, C. E. V., Voigt, B., Henry, J. D., Rendell, P. G., \& Aberle, I. (2013). The development of prospective memory in young school children: The impact of ongoing task absorption, cue salience, and cue centrality. Journal of Experimental Child Psychology, 116, 792-810.

Kvavilashvili, L., Kyle, F., \& Messer, D. J. (2008). The development of prospective memory in children: Methodological issues, empirical findings, and future directions. In M. Kliegel, M. A. McDaniel, \& G. O. Einstein (Eds.), Prospective memory: Cognitive, neuroscience, developmental, and applied perspectives (pp. 115-140). Mahwah, NJ: Lawrence Erlbaum.

Kvavilashvili, L., Messer, D. J., \& Ebdon, P. (2001). Prospective memory in children: The effects of age and task interruption. Developmental Psychology, 37, 418-430.

Mahy, C. E. V., \& Moses, L. J. (2011). Executive functioning and prospective memory in young children. Cognitive Development, 26, 269-281.

Mahy, C. E. V., \& Moses, L. J. (2013, April). The role of cognitive monitoring in children's remembering. In Symposium session presented at the biennial meeting of the Society for Research in Child Development, Seattle, WA.

Marcovitch, S., Boseovski, J. J., \& Knapp, R. J. (2007). Use it or lose it: Examining preschoolers' difficulty in maintaining and executing a goal. Developmental Science, 10, 559-564.

Martin, M., Kliegel, M., \& McDaniel, M. A. (2003). The involvement of executive functions in prospective memory performance of adults. International Journal of Psychology, 4, 195-206.

Maylor, E. A. (1996). Does prospective memory decline with age? In M. Brandimonte, G. O. Einstein, \& M. A. McDaniel (Eds.), Prospective memory: Theory and applications (pp. 173-197). Mahwah, NJ: Lawrence Erlbaum.

McDaniel, M. A., \& Einstein, G. O. (2000). Strategic and automatic processes in prospective memory retrieval: A multiprocess framework. Applied Cognitive Psychology, 14, S127-S144.

McGann, D., Defeyter, M. A., Ellis, J. A., \& Reid, C. (2005, July). Prospective memory in children: The effects of age and target salience. In Paper presented at the Second International Conference on Prospective Memory, Zurich, Switzerland.

Meacham, J. A. (1982). A note on remembering to execute planned actions. Journal of Applied Developmental Psychology, 3, 121-133.

Miyake, A., Friedman, N. P., Emerson, M. J., Witzki, A. H., Howerter, A., \& Wager, T. D. (2000). The unity and diversity of executive functions and their contributions to complex "frontal lobe" tasks: A latent variable analysis. Cognitive Psychology, 41, 49-100.

Otani, H., Landau, J. D., Libkuman, T. M., Louis, J. P. S., Kazen, J. K., \& Throne, G. W. (1997). Prospective memory and divided attention. Memory, 5, 343-360.

Perner, J., \& Lang, B. (1999). Development of theory of mind and executive control. Trends in Cognitive Sciences, 3, $337-344$. 
Preacher, K. J., \& Hayes, A. F. (2008). Asymptotic and resampling strategies for assessing and comparing indirect effects in multiple mediator models. Behavior Research Methods, 40, 879-891.

Rueda, M. R., Rothbart, M. K., McCandliss, B. D., Saccomanno, L., \& Posner, M. I. (2005). Training, maturation, and genetic influences on the development of executive attention. Proceedings of the National Academy of Sciences of the United States of America, 102, 14931-14936.

Schnitzspahn, K. M., Stahl, C., Zeintl, M., Kaller, C. P., \& Kliegel, M. (2013). The role of shifting, updating, and inhibition in prospective memory: Performance in young and older adults. Developmental Psychology, 49, 1544-1553.

Somerville, S. C., Wellman, H. M., \& Cultice, J. C. (1983). Young children's deliberate reminding. Journal of Genetic Psychology, 143(595), 87-96.

Smith, R. E. (2003). The cost of remembering to remember in event-based prospective memory: Investigating the capacity demands of delayed intention performance. Journal of Experimental Psychology: Learning, Memory, and Cognition, 29, 347-361.

Wang, L., Kliegel, M., Liu, W., \& Yang, Z. (2008). Prospective memory performance in preschoolers: Inhibitory control matters. European Journal of Developmental Psychology, 5, 289-302.

Ward, H., Shum, D., McKinlay, L., Baker-Tweney, S., \& Wallace, G. (2005). Development of prospective memory: Tasks based on the prefrontal-lobe model. Child Neuropsychology, 11, 527-549.

Wechsler, D. (1991). Wechsler intelligence scale for children-Third edition. San Antonio, TX: Psychological Corporation.

Zelazo, P. D., Carlson, S. M., \& Kesek, A. (2008). The development of executive function in childhood. In C. A. Nelson \& M. Luciana (Eds.), Handbook of developmental cognitive neuroscience (2nd ed., pp. 553-574). Cambridge, MA: MIT Press.

Zelazo, P. D., Carter, A., Reznick, J. S., \& Frye, D. (1997). Early development of executive function: A problem-solving framework. Review of General Psychology, 1, 198-226.

Zelazo, P. D., Müller, U., Frye, D., \& Marcovitch, S. (2003). The development of executive function in early childhood. Monographs of the Society for Research on Child Development, 68(3, Serial No. 274). 